\title{
Frustración, temperamento y vulnerabilidad social en bebés: estudio comparativo
}

\section{Frustration, temperament and social vulnerability in babies: a comparative study}

\author{
Mery Hernández Escalona ${ }^{\mathrm{a}, *}$, Angel M. Elgier ${ }^{\mathrm{a}, \mathrm{b}, \mathrm{c}}$, Lucas G. Gago Galvagno ${ }^{\mathrm{a}, \mathrm{b}, \mathrm{c}}$, Alba E. Mustaca ${ }^{\mathrm{b}}$ \\ ${ }^{a}$ Facultad Latinoamericana de Ciencias Sociales, Argentina \\ bUniversidad Abierta Interamericana, Argentina

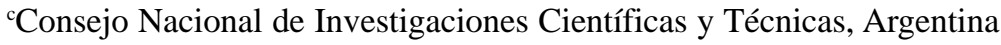

\section{Resumen}

Antecedentes: la frustración se define como las respuestas del organismo que se desencadenan cuando existe una discrepancia negativa entre un incentivo esperado con el que realmente se recibe. Estas situaciones provocan respuestas conductuales, emocionales y neurofisiológicas análogas a las que ocurren con la presentación de estímulos aversivos o su anticipación. Existen investigaciones en bebés, pero pocos sobre sus asociaciones con diferencias individuales y ambientales y ninguno en población argentina. Objetivos: asociar la frustración, el temperamento y la vulnerabilidad social en una muestra de infantes. Metodología: se realizó un estudio asociativo-comparativo con una situación observacional estructurada donde se evaluaron a 22 bebés de 10 a 14 meses durante una tarea de frustración y su relación con el temperamento a través del Cuestionario de Conducta Infantil (IBQ-VSF) pertenecientes a dos extractos sociales evaluados con una ficha sociodemográfica. Resultados: se halló que a mayor extroversión, menor manipulación del objeto; y a mayor esfuerzo de control, menor fue la respuesta de morder, y viceversa. No se encontraron diferencias significativas en función del nivel socioeconómico. Conclusiones: se discutieron los resultados en función de la teoría de la frustración y las limitaciones del estudio, incluyendo recomendaciones para futuras investigaciones.

Palabras clave: frustración; temperamento; vulnerabilidad social; infancia.

\section{Para citar este artículo:}

Hernández, M., Elgier, A. M., Gago, L. G., \& Mustaca, A. E. (2021). Frustración, temperamento y vulnerabilidad social en bebés: estudio comparativo. Liberabit, 27(1), e403. https://doi.org/10.24265/ liberabit.2021.v27n1.06

\begin{abstract}
Background: Frustration is defined as the body's responses that are triggered when there is a negative discrepancy between an expected incentive and the one actually received. These situations elicit behavioral, emotional and neurophysiological responses analogous to those that occur with aversive stimuli or their anticipation. There is research on babies, but few on the associations with individual and environmental differences and none on the Argentine population. Objectives: To associate frustration, temperament and social vulnerability in a sample of infants. Method: A comparative associative study was carried out using a structured observation technique where frustration and its relationship with temperament were evaluated in 22 babies aged 10 to 14 months through the infant behavior questionnaire-revised very short form (IBQ-R VSF). The study population belonged to two social strata according to a sociodemographic record. Results: An inverse correlation was found between extroversion and manipulation of objects, and between effortful control and biting response. No significant differences were identified based on the socioeconomic level. Conclusions: The results were discussed taking into account the frustration theory, the study limitations and the recommendations for future research.
\end{abstract}

Keywords: Frustration; temperament; social vulnerability; infancy.

Este es un artículo Open Access publicado bajo la licencia Creative Commons Atribución 4.0 Internacional. (CC-BY 4.0)

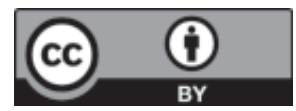

Universidad de San Martín de Porres, Lima - Perú http://ojs3.revistaliberabit.com 


\section{Introducción}

Hoy en día existe cierta discrepancia en cuanto a la concepción y naturaleza de las emociones. Las teorías contemporáneas han reconocido la importancia de conocerlas y manejarlas como parte fundamental del desarrollo del individuo, ya que, además de favorecer las habilidades sociales, promueven al desarrollo de competencias cognitivas (Bennett et al., 2005; Garner \& Waajid, 2012) y del lenguaje (Garner \& Waajid, 2008) desde edades muy tempranas. Andrés (2014) señala que, a pesar de las diferencias en los planteos relacionados a las emociones, existe un acuerdo al considerarlas como aquellas reacciones del organismo necesarias para su adaptación al entorno, que se presentan como el resultado de una evaluación cognitiva en la que intervienen aspectos atencionales y de regulación, que a su vez genera una activación de los componentes comportamentales, fisiológicos y subjetivos del individuo, lo que permite el autocontrol emocional.

Se ha mostrado que los individuos desde edades muy tempranas pueden manifestar emociones. Esto hace presumir que los seres humanos, desde el momento de su nacimiento, se encuentran dotados de un sistema biológicamente exitoso que permite su reconocimiento, además de mostrar cambios conductuales relacionados con ellas (Andrés, 2014; Barch et al., 2019; Kobre \& Lipsitt, 1972; Nelson et al., 2019).

El desarrollo de las emociones está modulado tanto por factores ambientales como individuales, aunque ambos interaccionan constantemente. Entre los factores individuales se consideran la edad, el sexo, el desarrollo de habilidades cognitivas (Bennett et al., 2005), la maduración del cerebro y redes atencionales, la capacidad motora, la competencia lingüística y el temperamento (Brandes-Aitken et al., 2019; Ato et al., 2004), entre otros. Entre las variables ambientales se destacan la interacción padre-hijo (Brophy et al., 2012; Cimino et al., 2016; Kennedy \& Rubin, 2010), las pautas de crianza y del lenguaje afectivo usado por los padres (Brophy et al.,
2012; Ato et al., 2004), la relación docente-alumno (Garner \& Waajid, 2008) y el nivel socioeconómico (Bradley \& Corwyn, 2002; Gago et al., 2019).

El temperamento se define como las diferencias individuales en la reactividad y la autorregulación, que tienen un origen constitucional por su dotación genética (Rothbart, 2011), aunque se encuentra regulado por el medio ambiente y por el aprendizaje (Rothbart et al., 2000), determina las respuestas afectivas, atencionales y motoras en diferentes situaciones y tiene un rol en las interacciones sociales subsecuentes y en el funcionamiento social (Posner \& Rothbart, 2018; Rothbart, 2011). Los estilos temperamentales descriptos por Rothbart et al. (2000) se clasifican en dimensiones: la extroversión, definida como la disposición hacia emociones positivas, tendencia al acercamiento hacia refuerzos y a un alto nivel de actividad; el afecto negativo, que incluye las dimensiones relacionadas con el miedo, la rabia, la tristeza, la irritabilidad/malestar y la falta de capacidad para calmarse; y, por último, el esfuerzo de control, que corresponde al componente regulatorio del temperamento e incluye el cambio de atención y enfoque, la sensibilidad perceptual, la expresión del placer de baja intensidad (cuando el niño experimenta placer en actividades tranquilas como jugar con su juguete favorito) y el control inhibitorio (Mira \& Nuñez, 2017; Rothbart, 2011).

La vulnerabilidad social se concibe como una variable multidimensional que comprende no solo el ingreso económico, sino también el nivel educativo de la familia, el tipo de ocupación, las características del hogar y el acceso al sistema de salud, entre otros (Brugué et al., 2018). Son varias las investigaciones que muestran que la vulnerabilidad social se relaciona con el déficit en las habilidades cognitivas de autorregulación durante la primera infancia (Gago et al., 2019; Kia-Keating et al., 2018); ya que involucra, entre algunos aspectos, cuestiones vinculadas con los estilos de crianza autoritarios o negligentes (Rubilar et al., 2017), exposición a estrés, violencia y bajos niveles nutricionales y de salud (Meléndez \& Solano, 
2017), que van en detrimento de un desarrollo adecuado en estas etapas del ciclo vital (Hernández et al., 2019). En otras pruebas sobre el desarrollo cognitivo y emocional, se halló que los infantes que provienen de extractos socioeconómicos bajos tienden a mostrar mayor tiempo de reacción en las tareas de atención, alerta y orientación, asociándose a puntajes más bajos en los informes de sus madres en esfuerzo voluntario de control (e.g., Lipina et al., 2004; Lipina \& Colombo, 2009; Putnam \& Rothbart, 2006). Sin embargo, otros artículos fallaron en encontrar relaciones entre los niveles socioeconómicos y el desarrollo cognitivo y emocional de los infantes. Esto puede deberse a varios factores, por ejemplo, diferentes métodos para evaluar los niveles socioeconómicos, que se tomen grupos con discrepancias socioeconómicas pequeñas y otras muy dispares y pruebas poco sensibles, entre otros.

Una de las emociones que se manifiestan frecuentemente es la frustración. Se define como la reacción emocional, cognitiva, fisiológica y neural del organismo que se desencadena cuando existe una discrepancia negativa entre un incentivo esperado con el que realmente se recibe. La pérdida de seres queridos, salir aplazado en un examen cuando se presumía que iba a ser aprobado con muy buena nota o ser despedido de un trabajo son ejemplos de eventos de frustración. Se ha mostrado que la frustración desencadena respuestas inmediatas incondicionadas conductuales, emocionales, fisiológicas y neurofisiológicas análogas a las que provocan la presentación de estímulos aversivos o su anticipación, tales como la ansiedad, el miedo, el estrés y el dolor; ellas son transitorias, aunque sus consecuencias pueden prolongarse (Mustaca, 2013, 2018).

En los humanos, la respuesta de frustración se manifiesta prácticamente desde el nacimiento, de lo que se infiere que nacen dotados de un sistema que permite tener expectativas acerca del futuro en función de memorias del pasado y tener respuestas diferenciales medibles si no se cumple lo que se espera. Uno de los primeros antecedentes fue el realizado por Kobre y Lipsitt (1972). Hallaron que bebés de 4 horas dejaron abruptamente de consumir agua de un biberón si previamente recibieron agua azucarada, a diferencia de un grupo de control que siempre recibía agua.

La frustración en bebés y niños se estudió mediante la devaluación, omisión o inaccesibilidad inesperada o sorpresiva de un reforzador positivo esperado por aprendizajes previos o por la situación. La manera de estudiarlos en la mayoría de los estudios tiene al menos dos fases. En la primera, se permite al infante manipular o recibir incentivos durante un tiempo estipulado (e.g., alimentos, juguetes, películas, atención social) para generar emociones placenteras. En una segunda fase (de frustración), se le quita, aleja o disminuye el incentivo; a veces, se presenta una tercera fase donde el niño vuelve a recuperarlo. Se evalúan principalmente las respuestas de la segunda fase. Las conductas observables ante la violación de expectativas son reacciones negativas que cambian en relación a su etapa evolutiva, por ejemplo, llanto, pataletas, evitación, actividad motora, aumento del eje adrenocortical, aumento del ritmo cardíaco, uso de verbalizaciones, etc. (Kamenetzky et al., 2009; Hernández et al., 2019). A medida que transcurre el tiempo, las respuestas serán más controladas, puesto que habrá desarrollado mecanismos de regulación, aunque la primera reacción serán siempre respuestas emocionales negativas (e.g., Braungart \& Stifter, 1996; Fox \& Calkins, 2003). La intensidad de estas reacciones está determinada por la discrepancia entre el incentivo esperado y el recibido y con la motivación, por ejemplo, a mayor exposición e interés por el incentivo presentado, mayor será la intensidad de las reacciones negativas. Estos resultados son similares a los hallados en otras especies y parece estar vinculado a las leyes generales de la percepción (Papini \& Pellegrini, 2006). Los aprendizajes previos también influyen en estas respuestas; principalmente, los programas de reforzamiento parcial logran disminuir las respuestas negativas y aumentar la perseverancia durante la segunda fase (e.g., 
Pellegrini et al., 2004). Además, están influenciadas por características individuales, entre ellas el temperamento. Los niños que tienden a ser más reactivos ante situaciones inesperadas, suelen manifestar más expresiones de enojo, llanto, pataletas, son más distraídos, con foco atencional más dispersos y tienen una tasa cardíaca más alta que los niños menos reactivos. Estas diferencias estarían mediadas por la posibilidad de regular las emociones (e.g., Gago Galvagno et al., 2019; Hernández et al., 2019). En la mayoría de los estudios no se observaron diferencias en función del sexo de los bebés.

Respecto a la influencia del nivel socioeconómico en pruebas de frustración, se halló un trabajo con resultados negativos en niños de 6 meses (Calkins et al., 2002). Otras investigaciones hallaron que el temperamento y el nivel socioeconómico regulaban la intensidad y duración de la afectividad positiva y negativa durante la fase II del Paradigma Still-Face en el cual hay una supresión abrupta de un reforzador social, ya que la madre deja de interactuar con el infante durante dos minutos (Gago et al., 2019; Smaling et al., 2016). Específicamente, estos autores encontraron que a mayor nivel educativo de los padres, ingreso económico y esfuerzo de control reportado, las conductas negativas (i.e., gritar, sollozar) y la aversión a la mirada a la madre durante la prueba Still-Face tendían a disminuir, y las conductas de emocionalidad positiva (i.e., sonrisas, risas) aumentaban. A resultados similares arribaron Gago et al. (2020) en su estudio donde encontraron que el hacinamiento tendía a incrementar las conductas negativas y agresivas durante la prueba Still-Face.

En esta publicación se estudiará la respuesta de frustración en bebés y la influencia de sus diferencias individuales (el temperamento y el género) y del ambiente, pertenecientes a hogares con necesidades básicas satisfechas (NBS) y con necesidades básicas insatisfechas (NBI). Si bien hubo trabajos previos sobre esta emoción en esa etapa del desarrollo, muy pocos la compararon con diferencias en el nivel socioeconómico de su entorno y ninguno fue realizado en poblaciones con vulnerabilidad social.

En función de los antecedentes presentados, esta investigación tuvo como objetivos: a) evaluar las conductas de bebés de entre 10 a 14 meses de edad ante una tarea irresoluble (acceder a un juguete inaccesible con el que anteriormente había jugado), y b) identificar si ellas se diferencian en función del temperamento reportado por la madre (y/o padre) y del nivel socioeconómico familiar. De acuerdo a los antecedentes teóricos, se predice que el temperamento influirá en sus respuestas; los infantes con menores niveles de afectividad negativa y mayor esfuerzo de control mostrarán menor intensidad de respuestas emocionales. En relación a la vulnerabilidad social, aunque se carece de antecedentes en esta tarea, se presume que los bebés pertenecientes a hogares con necesidades básicas satisfechas (NBS) mostrarán comportamientos más regulados en comparación con los provenientes de hogares con necesidades básicas insatisfechas (NBI). Así, el propósito general de esta investigación fue conocer los mecanismos y factores individuales y ambientales que influyen en la reacción de los bebés ante eventos frustrantes.

\section{Método}

\section{Diseño}

La presente investigación se trata de un estudio asociativo comparativo, con una situación observacional estructurada, de acuerdo a la clasificación de Ato et al. (2013).

\section{Muestra}

El tipo de muestreo fue no probabilístico por conveniencia; estuvo conformado por 22 díadas madre-bebé argentinos con edades comprendidas entre 10 a 14 meses $(M=12.46, D E=1.41)$, de los cuales 14 (8 varones y 6 mujeres) provenían de hogares con NBS y 8 (7 varones y 1 mujer) eran de hogares con NBI. Ambos grupos concurrieron a una sala de atención primaria de salud de la localidad de 
San Miguel, provincia de Buenos Aires, y a los consultorios de control de salud de un Hospital de Niños de la Ciudad Autónoma de Buenos Aires, Argentina.

La selección de la muestra persiguió los siguientes criterios: español como idioma nativo, visión y audición normales, sin evidencia de enfermedad grave, sin antecedentes familiares de enfermedad psiquiátrica, sin antecedentes de lesiones, sin convulsiones o enfermedades neurológicas, y sin abuso o dependencia de sustancias por parte de la madre. Los bebés no debían presentar síntomas de enfermedad aguda, nacer a término y con la altura y el peso adecuados para su edad. Para esto, se revisaron las historias clínicas de la madre y los bebés, además de la información de la madre en la escala de nivel económico social (NES) que se presentará más adelante.

\section{Instrumentos}

Cuestionario de Comportamiento Infantil (Infant Behavior Questionnaire, IBQ-VSF; Putnam et al., 2014). Para la evaluación del temperamento se administró la versión corta de este cuestionario para infantes de 3 a 12 meses. Fue traducido al castellano por el Grupo de Investigación en Psicología Evolutiva de la Universidad de Murcia (España) y utilizado en estudios previos (Gutiérrez et al., 2018). Los análisis factoriales del cuestionario realizados indican que evalúa, entre otros, los siguientes procesos cognitivos: nivel de actividad, sonrisa, miedo, angustia o enojo a las limitaciones, orientación y calma de los niños (Rothbart \& Rueda, 2005). Tiene 36 ítems que evalúa tres dimensiones temperamentales: a) extroversión (13 ítems), se caracteriza por altos niveles de actividad, sociabilidad, impulsividad y posibilidad de disfrutar de momentos de alta intensidad de placer; b) afectividad negativa (12 ítems), caracterizado por miedo, enojo o frustración, disconformidad y tristeza; y c) esfuerzo de control (10 ítem), que describe comportamientos relacionados con el desarrollo autorregulatorio, tanto emocional como cognitivo (Pesonen et al., 2006; Rothbart \& Posner, 2005). Este último se caracteriza por altos niveles de concentración, control inhibitorio, facilidad para mover el foco atencional, sensibilidad perceptual y baja intensidad de placer. La evaluación la hacen los padres de los niños. Ellos debían responder si la conducta que describía cada ítem ocurrió durante la última semana previa a la administración del cuestionario mediante una escala Likert de 8 puntos que indicaba: (1) nunca, (2) muy raramente, (3) pocas veces, (4) la mitad de las veces, (5) muchas veces, (6) casi siempre, (7) siempre, (X) no sucedió en la semana (Rothbart \& Gartstein, 2003). La puntuación de cada una de las tres dimensiones representó la puntuación media de los ítems incluidos en ella. Es decir, se suman los puntajes de cada dimensión (la X indica puntaje 0 ) y se divide por el número de ítems correspondientes. Las puntuaciones más altas indican niveles más altos en el factor evaluado. En el estudio de Putnam et al. (2014) la escala presentó acuerdo interpadres similar al obtenido en la versión revisada de este cuestionario, siendo en promedio de .41. Por otro lado, la estabilidad longitudinal y la confiabilidad test-retest tuvo un rango de .54 a .93. En cuanto a la validez convergente y predictiva de observaciones comportamentales fue adecuada y similar a la versión revisada. La escala presentó un alfa de Cronbach mayor a .70 para sus subescalas. En este estudio, el alfa osciló entre .65 y .72 para las subescalas. Este cuestionario aún no tiene validación en una población de Argentina.

\section{Evaluación de la situación socioeconómica} familiar. Para obtener las características socioeconómicas y ambientales de los hogares de los bebés que participaron en el estudio se administró, a través de una entrevista con las madres, una ficha de exploración de características sociodemográficas que ya ha sido utilizada en investigaciones previas (Elgier et al., 2017; Gago et al., 2019). Se basa en los criterios de medición de la pobreza utilizados por el Instituto Nacional de Estadística y Censos (INDEC, 2001). Las variables que se tuvieron en cuenta fueron la información referida a la salud pre, peri y postnatal del bebé como, por ejemplo, cantidad de controles médicos que se realizaron durante el 
embarazo, peso, talla y perímetro cefálico del bebé al nacer, pérdida de conocimiento a raíz de golpes en la cabeza, operaciones e intervenciones prolongadas. La valoración de este ítem estuvo comprendida entre presencia («sí») o ausencia («no»), con algunas respuestas específicas cuando la pregunta lo requiriese (ejemplo, pesos del bebé al nacer). La información obtenida permitió conocer la ausencia o presencia de alguna patología o trastorno en el bebé. Los criterios tomados para identificar a los hogares con NBI fueron los que presentaron al menos uno de los siguientes indicadores de privación: a) hacinamiento, hogares con más de tres personas por cuarto; b) tipo de vivienda, pieza de inquilinato, casillas, hotel o pensiones, casas tomadas, viviendas precarias (e.g., con piso de tierra, paredes de cartón o chapa, provisión de agua de pozo, sin baño propio); c) condiciones sanitarias, hogares que no tuvieran ningún tipo de retrete (sin descarga cloacal o falta de inodoro en el baño); d) menores en edad escolar que no asisten a la escuela, hogares que tuvieran algún infante en edad escolar (6 a 18 años) que no asistiera a la escuela; y e) competencia laboral del jefe de hogar, hogares que tuvieran cuatro o más personas por miembro ocupado y, además, cuyo jefe no hubiera completado la escolaridad primaria. En cuanto a los hogares con NBS, se consideraron aquellos en los que no se encontraba presente ninguno de los indicadores mencionados anteriormente.

Evaluación de la frustración. La situación de frustración fue una parte de varias evaluaciones que se le realizaron al bebé previamente. Se desarrolló dentro de un contexto lúdico con un conjunto de juegos. El bebé, la madre y el investigador estaban sentados en el suelo sobre una alfombra con los juguetes; la mamá estaba detrás del bebé, mientras que el investigador se sentaba enfrente con el fin de presentarle los juguetes y moderar las actividades. La cámara estaba dispuesta en uno de los extremos del área donde se trabajó, la manejaba otro experimentador que se encargaba de filmar las situaciones y las conductas del infante. La parte de la filmación que se consideró para este estudio consistió en dos fases. En la primera, se le dio al bebé un auto de juguete que manipuló entre 60 a 90 segundos. En la fase 2, el investigador le retira el juguete, lo introduce dentro de un envase transparente de plástico y lo tapa de modo que no lo pueda abrir y se lo vuelve a entregar. En esta etapa se inicia la observación y medición de las conductas del bebé. El tiempo de duración de la situación completa fue de un minuto y medio aproximadamente; se consideró terminada la sesión cuando el bebé abandonaba el juguete, lo tiraba lejos o se alejaba de él. La fase dos es considerada como una situación de frustración debido a que se permitió al infante jugar con un cochecito (incentivo o reforzador positivo), que luego de manera inesperada es retirado de su alcance para introducirlo en una caja y dársela sin posibilidad de abrirla y seguir jugando con el juguete.

Los comportamientos fueron grabados y cronometrados. Para ello se utilizó una grabadora de video Sony HD HDR-CX160® y un cronómetro Modelo CR202 de la línea Galileo Italy®.

\section{Codificación y análisis del material observacional}

Para categorizar las respuestas se tuvieron en cuenta las investigaciones previas (Calkins et al., 2002). Para la obtención de confiablidad de las medidas, las observaciones fueron medidas dos veces por el mismo experimentador a ciegas. Para las conductas con nivel de medición cuantitativo continua, el coeficiente intraclase fue mayor a .89.

Las conductas a observar se midieron a ciegas (sin saber a qué grupo pertenecían los bebés), previo acuerdo entre los investigadores para definirlas operacionalmente con la mayor precisión. Estas fueron las siguientes:

a) Tasa de manipulación del envase desde que lo toca hasta que lo deja de tocar (en segundos). A mayor manipulación, mayor perseverancia. 
b) Tipo de manipulación del objeto (frecuencia): sacudir, golpear, darle vuelta y morderlo. Sugiere intento de abrir el envase.

c) Juego simbólico (frecuencia): arrastrar el envase como si fuera un auto. Esta conducta se considera dentro de respuestas de regulación de emocional.

d) Duración de mirada al adulto (medida en segundos). Indica una solicitud de ayuda ante la imposibilidad de resolver el problema; se considera una forma de regulación emocional.

e) Balbuceo del bebé (frecuencia).

f) Llanto del bebé (frecuencia). A mayor cantidad de veces, mayor intensidad de la reacción emocional de frustración.

g) Alejamiento del bebé del envase (frecuencia): tira lejos el envase o se aleja de él gateando. Se considera una reacción emocional de frustración o de abandono de la tarea (extinción).

\section{Procedimiento y análisis de datos}

La recolección de datos se realizó en espacios dispuestos por las instituciones de salud. En una primera instancia, se convocó a una reunión a los padres de los bebés que asistían a la sala y consultorios de lactantes, tras la autorización del personal directivo y del centro de salud. En la entrevista se les comunicaron los objetivos del trabajo, así como las tareas y procedimientos que fueron necesarios para la evaluación del bebé. Se explicó que todas las tareas se realizarían en presencia de la madre, siendo necesaria su participación activa en algunas de ellas. Se les informó acerca del consentimiento informado y de confidencialidad de los datos de los padres y del infante y se aclaró que podrían dejar de participar en cualquier etapa de la evaluación. Con aquellos padres que aceptaron participar en el estudio, se programaron entrevistas individuales con el fin de desarrollar las evaluaciones en presencia de ellos. En todos los casos durante la evaluación de los bebés participaron las madres. Después de culminadas las observaciones, se prosiguió a entrevistar a las madres con el fin de administrar la escala de Nivel Económico Social (NES) y el cuestionario de conducta infantil (Infant Behavior Questionnaire, [IBQ-VSF]). Para la tarea que fue evaluada en esta investigación, se le solicitó a la madre que, durante su desarrollo, tuviera una postura neutra.

Los resultados se analizaron con el programa SPSS versión 24. Dado el reducido número de participantes, se realizaron pruebas inferenciales no paramétricas, específicamente, la prueba $U$ de MannWhitney y Rho de Spearman. Se estableció un criterio de significación de $p<.05$.

\section{Resultados}

Una observación asistemática de las respuestas de los bebés durante la prueba de frustración indica que casi todas ellas tuvieron secuencias similares: el bebé toma el envase, lo manipula, lo observa, mira al adulto, se lo pasa como para que lo tome, realizan un juego simbólico, como, por ejemplo, arrastrar la caja como si fuera el cochecito, y, finalmente, lo deja cambiando el foco atencional tirándolo o alejándose de él. No se observaron gestos de enojo o irritabilidad, a excepción de dos bebés que lloraron.

En cuanto a los valores cuantitativos, predominaron las conductas (medidas en segundos) de manipulación del objeto $(M=36.6, D E=14.43)$. Un bebé de cada grupo social lloró con baja intensidad y tres de NBI y nueve de NBS se alejaron del juguete en varias oportunidades (tirándolo o alejándose gateando) para luego volver a manipularlo. Por otra parte, 3 bebés NBI y 2 NBS realizaron conductas de juego simbólico. El análisis de contraste de grupos revela ausencia de diferencias significativas en función del nivel socioeconómico, edad y género.

En relación con el temperamento, se observa que los bebés NBS obtienen un mayor puntaje en extroversión y menor en afectividad negativa en 
comparación con los de NBI. En esfuerzo de control los valores son prácticamente similares. Sin embargo, el análisis de contraste indica que las diferencias no son significativas en ninguna de las dimensiones ni en función del sexo.

Por último, se efectuó una correlación entre las medidas comportamentales y las dimensiones del temperamento. Se encontraron asociaciones negativas y significativas entre los puntajes de extroversión y la duración de la manipulación del objeto $(r=-.49, p$ $=.04$ ), y entre el esfuerzo de control con la respuesta de morder el objeto $(r=-.51, p=.03)$. Esto indica que, a mayor extroversión, menor tiempo de manipulación del objeto, y a mayor esfuerzo de control, menor es la respuesta de morder.

\section{Discusión}

Los objetivos de esta investigación fueron evaluar las conductas de bebés entre 10 a 14 meses de edad ante una situación de frustración (acceder a un juguete inaccesible, con el que anteriormente habían jugado) y evaluar la asociación de esas conductas en función de su temperamento y nivel socioeconómico.

Las conductas más frecuentes que se observaron fueron la manipulación del objeto, mirada al adulto y alejamiento del envase. Esto podría deberse a que las interacciones madre-infante en esta edad se dan a partir de conductas comunicativas no verbales, la manipulación de objetos y la mirada hacia el adulto (Adamson et al., 2019). Esto posibilita que el infante pueda realizar intercambios con el adulto e intento que su entorno lo ayude (Adamson et al., 2019; Gago et al., 2019).

Los resultados negativos obtenidos de la frustración en relación al nivel socioeconómico de los hogares de los niños son semejantes a lo obtenido por Calkins et al. (2002), aunque contrarios a otros estudios (Andraca et al., 1998; Farah et al., 2006; Gago et al., 2019, 2020; Smaling et al., 2016). Por ejemplo, Andraca et al. (1998) mostraron la influencia perjudicial que ejerce el bajo nivel de estimulación en hogares desfavorables sobre desarrollo cognitivo y motor en los bebés. Del mismo modo, la revisión hecha por Mazzoni et al. (2014) destaca la consecuencia negativa que ejercen los ambientes con pocos estímulos sobre el desarrollo cognitivo del infante. Por otra parte, Farah et al. (2006), Gago et al. (2019, 2020) y Smaling et al. (2016), hallaron que los bebés que provienen de nivel socioeconómico bajo tienden a tener un desarrollo del lenguaje y regulación emocional menor en comparación a los infantes pertenecientes a clase media. Hay que hacer notar que, en estas últimas investigaciones, los niños fueron estudiados en tareas diferentes a una situación de frustración. Sin embargo, en el Paradigma de StillFace se hallaron diferencias entre niños de niveles socioeconómicos distintos. Una posible interpretación sobre los resultados que hallamos en relación al nivel socioeconómico es que en la frustración las respuestas inmediatas que predominan son incondicionadas y muy poco influidas por el ambiente.

Por otro lado, se encontraron dos correlaciones negativas entre los estilos temperamentales y las conductas evaluadas. Los niños con mayor puntaje en extroversión manipularon menos la caja con el juguete y los que obtuvieron menor puntaje en esfuerzo de control, lo mordieron más. Respecto a la primera relación, podría interpretarse que este estilo temperamental va de la mano con la interacción con adultos y búsqueda de sensaciones en infantes, por ende, a mayor extroversión tenderían a manipular menos el recipiente, ya que es una conducta realizada en solitario que podría implicar mayor grado de perseveración propia del temperamento más introvertido. La asociación negativa con el esfuerzo de control y la conducta de morder se podría interpretar de manera que los altos niveles de inhibición podrían disminuir las conductas emocionales negativas durante la prueba de frustración. Cabe aclarar que los índices de correlación fueron altos para este rango etario (Kochanska et al., 2000). A pesar de que se encuentran investigaciones que a estas edades existen asociaciones entre temperamento y habilidades cognitivas (Gago et al., 
2019; Posner \& Rothbart, 2018), en esta tarea se encontraron escasas asociaciones; se requieren más investigaciones con muestras locales.

Respecto a los datos obtenidos del temperamento en función al nivel socioeconómico al que pertenecían, si bien se obtuvo que el grupo NBS tuvo un mayor puntaje en extroversión y menor en afectividad negativa e igualdad de valores en esfuerzo de control, estos resultados no fueron significativos, por lo cual no se pudo determinar si el temperamento infantil podría tener algún tipo de influencia en las conductas de frustración en los bebés. Estos resultados se asemejan a los obtenidos por Reyna y Brussino (2015) que al evaluar, entre otros aspectos, la influencia entre el nivel socioeconómico sobre las habilidades sociales y conductuales en infantes en edad preescolar pudieron determinar que no existe un impacto significativo en su desarrollo. Asimismo, al comparar con los resultados del trabajo realizado por Prats et al. (2012), quienes evaluaron las relaciones entre el nivel socioeconómico (NBI-NBS) y el temperamento infantil, determinaron que ejercen una influencia parcial en su desarrollo emocional y cognitivo. Constataron que los infantes que vienen de hogares con necesidades insatisfechas, tuvieron un mayor tiempo de reacción en las tareas de atención, alerta y orientación. Ellas se asociaron a los puntajes más bajos de esfuerzo voluntario de control (temperamento) informados por sus padres. Si bien estos estudios no demuestran una estrecha relación entre el nivel socioeconómico de los niños y el temperamento infantil sobre su desarrollo cognitivo, se precisan de revisiones más profundas sobre los métodos usados para evaluar los niveles socioeconómicos, ya que tienden ser muy dispares entre los estudios.

La presente investigación tiene una serie de limitaciones propias de un estudio preliminar, por lo cual, se deben tomar los resultados con precaución; aun considerándolos útiles como para realizar nuevas investigaciones. Sin bien la ausencia de significación entre las variables puede deberse a que son respuestas incondicionadas y que por la edad puede no estar modulada por factores externos o individuales, se requieren más estudios controlados para llegar a esta conclusión debido a las limitaciones del estudio. El reducido número de participantes de la muestra pudo evitar hallar diferencias significativas entre las variables. Una segunda posibilidad es que la tarea no haya sido muy apropiada para evaluar la frustración. Si bien existen investigaciones donde hacen uso de juguetes sonoros y de colores llamativos (e.g., Braungart \& Stifter, 1996; Calkins et al., 2002; Mast et al., 1980; Stifter \& Grant, 1993), la diferencia en relación al presente estudio radica en que, a pesar de que la tarea consistió en darle el juguete al bebé, retirarlo de su alcance y luego regresárselo en una caja imposible de abrir, el tiempo inicial de manipulación pudo no ser suficiente como para generar reacciones intensas de frustración. Por otra parte, como el juguete se entregó encerrado, pudo traer como consecuencia que hayan predominado conductas más relacionadas con procesos cognitivos de resolver un problema (atencionales y de atención conjunta) y comunicativos, que con mecanismos de frustración como fueron las manipulaciones con el objetivo de abrir la caja y pedir ayuda a un adulto.

Las futuras investigaciones deberán sistematizar las actividades relativas a las medidas de frustración, así como estructurar mejor la tarea: dejar que el chico manipule el juguete por más tiempo y luego retirarlo de su alcance y dejarlo a la vista sin posibilidad de acceder a él para evitar que se solapen las posibles conductas cognitivas de resolución de problemas con las de frustración. La presencia de la madre o un adulto, durante el desarrollo de la tarea puede ser interesante para evaluar simultáneamente respuestas como atención conjunta o procesos de comunicación que permitan al bebé tener una regulación emocional a las respuestas típicas de frustración. También debería haber un ambiente con mayor control para aumentar su validez interna, como disponer de un espacio fijo, con menor estimulación visual, y, por supuesto, aumentar el número de participantes de la 
muestra. Finalmente, los estudios deberían seguir en el intento de comparar niños con hogares NBI y NBS, por el escaso número de publicaciones que abordan esta problemática en países con altos niveles de pobreza; ellos contribuirán a conocer más la posible influencia de la pobreza en las reacciones emocionales de los niños.

\section{Conflicto de intereses}

No existen conflictos de interés entre los autores del manuscrito.

\section{Responsabilidad ética}

El protocolo de este estudio fue aprobado por el Comité de Ética del Instituto de Investigaciones de la Universidad de Buenos Aires.

\section{Contribución de autoría}

MHE: recolección de datos, análisis estadístico, revisión final de manuscrito.

AE: concepción y diseño del estudio, recolección de datos.

LG: análisis estadístico, revisión final de manuscrito.

AM: concepción y diseño del estudio, revisión final de manuscrito.

\section{Referencias}

Adamson, L. B., Bakeman, R., Suma, K., \& Robins, D. L. (2019). An Expanded View of Joint Attention: Skill, Engagement, and Language in Typical Development and Autism. Child Development, 90(1), e1-e18. https:/ /doi.org/10.1111/cdev.12973

Andraca, I. D., Pino, P., La Parra, A. D., Rivera, F., \& Castillo, M. (1998). Risk factors for psychomotor development among infants born under optimal biological conditions. Revista de Saude Publica, 32(2), 138-147.
Andrés, M. L. (2014). Efecto mediador de las estrategias cognitivas de regulación emocional en la relación entre los rasgos de personalidad y la ansiedad, depresión y felicidad en niños de 9 a 12 años de edad [tesis de doctorado, Universidad Nacional de Mar del Plata]. Repositorio RPsico. http://rpsico.mdp.edu.ar/ handle/123456789/538

Ato, E., González, C., \& Carranza, J. (2004). Aspectos evolutivos de la autorregulación emocional en la infancia. Anales de Psicología, 20(1), 69-79.

Ato, M., López-García, J. J., \& Benavente, A. (2013). Un sistema de clasificación de los diseños de investigación en Psicología. Anales de Psicología, 29(3), 1695-2294. http://dx.doi.org/10.6018/analesps.29.3.178511

Barch, D. M., Harms, M. P., Tillman, R., Hawkey, E., \& Luby, J. L. (2019). Early Childhood Depression, Emotion Regulation, Episodic Memory, and Hippocampal Development. Journal of Abnormal Psychology, 128(1), 81-95. https://doi.org/10.1037/abn0000392

Bennett, D., Bendersky, M., \& Lewis, M. (2005). Antecedents of emotion knowledge: Predictors or Individual Differences in Young Children. Cognition and Emotion, 19(3), 375-396. https://doi.org/10.1080/ 02699930441000201

Bradley, R., \& Corwyn, R. (2002). Socioeconomic Status and Child Development. Annual Review of Psychology, 53, 371-399. https://doi.org/10.1146/ annurev.psych.53.100901.135233

Brandes-Aitken, A., Braren, S., Swingler, M., Voegtline, K., \& Blair, C. (2019). Sustained Attention in Infancy: A Foundation for the Development of Multiple Aspects of Self-Regulation for Children in Poverty. Journal of Experimental Child Psychology, 184, 192-209. https:/ /doi.org/10.1016/j.jecp.2019.04.006

Braungart, J. M., \& Stifter, C. A. (1996). Infants’ Responses to Frustrating Situations: Continuity and Change in Reactivity and Regulation. Child Development, 67(4), 1767-1779. https://doi.org/10.2307/1131730

Brophy, H., Stansbury, B., \& Horodynsky, M. (2012). Modeling Maternal Emotion-Related Socialization Behavior in a Low-Income Sample: Relations with Toddler's Self-Regulation. Early Childhood Research Quarterly, 27(3), 352-364. 
Brugué, Q., Gomà, R., \& Subirats, J. (2018). De la pobreza a la exclusión social. Nuevos retos para las políticas públicas. Revista Internacional de Sociología, 60(33), 7-45. https://doi.org/10.3989/ris.2002.i33.728

Calkins, S., Dedmon, S., Gill, K., Lomax, L., \& Johnson, L. (2002). Frustration in Infancy: Implications for Emotion Regulation, Physiological Processes, and Temperament. Infancy, 3(2), 175-197. https://doi.org/10.1207/ S15327078IN0302_4

Cimino, S., Cerniglia, L., Porreca, A., Simonelli, A., Ronconi, L., \& Ballarotto, G. (2016). Mothers and Fathers with Binge Eating Disorder and their 18-36 Months Old Children: A Longitudinal Study on Parent-Infant Interactions and Offspring's Emotional-Behavioral Profiles. Frontiers in Psychology, 7, 580. https:// doi.org/10.3389/fpsyg.2016.00580

Elgier, A. M., Galvagno, L. G., Clerici, G., Tortello, C., \& Azzolini, S. C. (2017). Seguimiento del gesto de señalar y de la mirada en estadios tempranos del desarrollo. Apuntes de Ciencia \& Sociedad, 7(1), 1120. https://doi.org/10.18259/acs.2017003

Farah, M., Shera, D., Savage J., Betancourt, L., Giannetta, J., Brodsky, N., Malmud, E., \& Hurt, H. (2006). Childhood Poverty: Specific Associations with Neurocognitive Development. Brain Research, 1110, 166-174.

Fox, N. A., \& Calkins, S. D. (2003). The development of self-control of emotion: Intrinsic and extrinsic influences. Motivation and Emotion, 27(1), 7-26.

Gago, L. G., De Grandis, M. C., Clerici, G. D., Mustaca, A. E., Miller, S. E., \& Elgier, A. M. (2019). Regulation during the Second Year: Executive Function and Emotion Regulation Links to Joint Attention, Temperament and Social Vulnerability in a Latin American Sample. Frontiers in Psychology, 10, 1473.

Gago, L. G., De Grandis, M. C., Jaume, L. C., \& Elgier, A. M. (2020). Home Environment and its Contribution to Early Childhood Regulatory Capabilities. Early Child Development and Care. https://doi.org/10.1080/ 03004430.2020.1796655

Garner, P., \& Waajid, B. (2008). The associations of emotion knowledge and teacher-child relationships to preschool children's school-related developmental competence.
Journal of Applied Developmental Psychology, 29(2), 89-100. https://doi.org/10.1016/j.appdev.2007.12.001

Garner, P., \& Waajid, B. (2012). Emotion Knowledge and Self-Regulation as Predictor of Preschoolers' Cognitive Ability, Classroom Behavior, and Social Competence. Journal of Psychoeducational Assessment, 30(4), 330343. https://doi.org/10.1177/0734282912449441

Gutiérrez, J., Celedón, V., Martínez, B., Rojas, F., Carmona, B., \& Farkas, C. (2018). Diferencias en temperamento según nivel socioeconómico en niños chilenos de 12 y 30 meses. Acta de Investigación Psicológica, 8(2), 42-58. https://doi.org/10.22201/fpsi.20074719e.2018.2.04

Hernández, M., Elgier, A. M., Gago, L. G., \& Mustaca, A. E. (2019). Emociones y frustración en bebés: el temperamento y la vulnerabilidad social como factores moduladores. Revista Subjetividad y Procesos Cognitivos, 23(2), 115-139.

Instituto Nacional de Estadística y Censos - INDEC. (2001). Incidencia de la pobreza en los aglomerados urbanos [boletín N. ${ }^{\circ}$ ]. INDEC.

Kamenetzky, G. V., Cuenya, L., Elgier, A. M., López Seal, F., Fosacheca, S., Martin, L., \& Mustaca, A. E. (2009). Respuestas de frustración en humanos. Terapia Psicológica, 27(2), 191-201. http://dx.doi.org/10.4067/ S0718-48082009000200005

Kennedy, K., \& Rubin, K. (2010). Gender and Parent's Reaction to Children's Emotion during the Preschool Years. New Directions for Child and Adolescent Development, 128, 51-64. https://doi.org/10.1002/cd.268

Kia-Keating, M., Nylund-Gibson, K., Kia-Keating, B. M., Schock, C., \& Grimm, R. P. (2018). Longitudinal Patterns of Self-Regulation among Ethnic Minority Children Facing Poverty. Journal of Child and Family Studies, 27(2), 398-411. https://doi.org/10.1007/s10826-0170883-5

Kobre, K. R., \& Lipsitt, L. P. (1972). A Negative Contrast Effect in Newborns. Journal of Experimental Child Psychology, 14(1), 81-91. https://doi.org/10.1016/00220965(72)90033-1

Kochanska, G., Murray, K. T., \& Harlan, E. T. (2000). Effortful Control in Early Childhood: Continuity and Change, Antecedents, and Implications for Social 
Development. Developmental Psychology, 36(2), 220232. https://doi.org/10.1037/0012-1649.36.2.220

Lipina, S. J., \& Colombo, J. A. (2009). Poverty and brain development during childhood: An approach from cognitive psychology and neuroscience. American Psychological Association.

Lipina, S. J., Martelli, M. I., Vuelta, B. L., Injoque-Ricle, I., \& Augusto, J. (2004). Pobreza y desempeño ejecutivo en alumnos preescolares de la ciudad de Buenos Aires (República Argentina). Interdisciplinaria, 21(2), 153193.

Mast, V. K., Fagen, J. W., Rovee-Collier, C. K., \& Sullivan, M. W. (1980). Immediate and Long-Term Memory for Reinforcement Context: The Development of Learned Expectancies in Early Infancy. Child Development, 51, 700-707. https://doi.org/10.2307/1129455

Mazzoni, C., Stelzer, F., Cervigni, M., \& Martino, P. (2014). Impacto de la pobreza en el desarrollo cognitivo. Un análisis teórico de dos factores mediadores. Liberabit, 20(1), 93-100.

Meléndez, L. M., \& Solano, V. S. (2017). La desnutrición y el estrés van a la escuela: pobreza infantil y neurodesarrollo en América Latina. Innovaciones Educativas, 19(27), 55-70.

Mira, A., \& Vera, L. (2017). Control esforzado: Componente regulatorio del temperamento y sus implicancias en el desarrollo socioemocional de los niños. Revista Chilena de Neuropsicología, 12(1), 24-28.

Mustaca, A. (2013). «Siento un dolor en el alma»: ¿metáfora o realidad? Revista Argentina de Ciencia del Comportamiento, 5(2), 47-60.

Mustaca, A. (2018). Frustración y conductas sociales. Avances en Psicología Latinoamericana, 36(1), 65-81. https://doi.org/10.12804/revistas.urosario.edu.co/apl/ a.4643

Nelson, M. B., O’Neil, S. H., Wisnowski, J. L., Hart, D., Sawardekar, S., Rauh, V., Perera, F., Andrews, H. F., Hoepner, L. A., Garcia, W., Algermissen, M., Bansal, R., \& Peterson, B. S. (2019). Maturation of Brain Microstructure and Metabolism Associates with Increased Capacity for Self-Regulation During the Transition from Childhood to Adolescence. Journal of
Neuroscience, 39(42), 8362-8375. https://doi.org/ 10.1523/JNEUROSCI.2422-18.2019

Papini, M. R., \& Pellegrini, S. (2006). Scaling relative incentive value in consummatory behavior. Learning and Motivation, 37(4), 357-378.

Pellegrini, S., Muzio, R., Mustaca, A., \& Papini, M. (2004). Successive Negative Contrast After Partial Reinforcement in the Consummatory Behavior of Rats Learning and Motivation. Learning and Motivation, 35(4), 303-321. https://doi.org/10.1016/j.lmot.2004.04.001

Pesonen, A. K., Räikkönen, K., Heinonen, K., Järvenpää, A. L., \& Strandberg, T. E. (2006). Depressive Vulnerability in Parents and Their 5-Year-Old Child's Temperament: A Family System Perspective. Journal of Family Psychology, 20(4), 648- 655. https://doi.org/ 10.1037/0893-3200.20.4.648

Posner, M. I., \& Rothbart, M. K. (2018). Temperament and Brain Networks of Attention. Philosophical Transactions of the Royal Society B: Biological Sciences, 373(1744), 20170254. https://doi.org/10.1098/ rstb.2017.0254

Prats, L. M., Fracchia, C. S., Segretin, S. M., Hermida, M. J., Colombo, J. A., \& Lipina, S. J. (2012). Predictores socioambientales e individuales del desempeño en una tarea atencional con demandas de alerta, orientación y control en niños de edad preescolar. Revista Argentina de Ciencias del Comportamiento, 4(2), 1931.

Putnam, S. P., \& Rothbart, M. K. (2006). Development of short and very short forms of the Children's Behavior Questionnaire. Journal of Personality Assessment, 87(1), 102-112.

Putnam, S. P., Helbig, A. L., Gartstein, M. A., Rothbart, M. K., \& Leerkes, E. (2014). Development and Assessment of Short and Very Short Forms of the Infant Behavior Questionnaire-Revised. Journal of Personality Assessment, 96(4), 445-458. https://doi.org/10.1080/ 00223891.2013 .841171

Reyna, C., \& Brussino, S. (2015). Diferencias de edad y género en comportamiento social, temperamento $\mathrm{y}$ regulación emocional en niños argentinos. Acta Colombiana de Psicología, 18(2), 51-64. http:// www.dx.doi.org/10.14718/ACP.2015.18.2.5 
Rothbart, M. K. (2011). Becoming who we are: Temperament and Personality in Development. Guilford Press.

Rothbart, M. K., \& Rueda, M. R. (2005). The Development of Effortful Control. En U. Mayr, E. Awh, \& S. Keele (Eds.), Developing Individuality in the Human Brain: Tribute to Michael I. Posner (pp. 167-188). American Psychological Association. https://doi.org/10.1037/ 11108-009

Rothbart, M. K., Ahadi, S. A., \& Evans, D. E. (2000). Temperament and Personality: Origins and Outcomes. Journal of Personality and Social Psychology, 78(1), 122-135. https://doi.org/10.1037/0022-3514.78.1.122

Rothbart, M., \& Gartstein, M. (2003). Studying Infant Temperament Via the Revised Infant Behavior Questionnaire. Infant Behavior \& Development, 26(1), 64-86. https://doi.org/10.1016/S0163-6383(02)00169-8
Rothbart, M. K., \& Posner, M. I. (2005). Genes and Experience in the Development of Executive Attention and Effortful Control. New Directions for Child and Adolescent Development, 109, 101-108. https://doi.org/ 10.1002/cd.142

Rubilar, J. V., Lemos, V., \& Richaud, M. C. (2017). Programa de fortalecimiento parental en contextos de vulnerabilidad social: Una propuesta desde el ámbito escolar. Interdisciplinaria, 34(1), 157-172.

Smaling, H., Huijbregts, S., Suurland, J., Heijden, K., Mesman, J., Goozen, S., \& Swaab, H. (2016). Prenatal Reflective Functioning and Accumulated Risk as Predictors of Maternal Interactive Behavior During Free Play, the Still-Face Paradigm, and Two Teaching Task. Infancy, 103, 1-19.

Stifter, C., \& Grant, W. (1993). Infant Responses to Frustration: Individual Differences in the Expression of Negative Affect. Journal of Nonverbal Behavior, 17(3), 187- 204. https://doi.org/10.1007/BF00986119 
Mery Hernández Escalona

Facultad Latinoamericana de Ciencias Sociales, Argentina.

Licenciada en Ciencias de la Educación, Universidad de los Andes (ULA). Magíster en Psicología Cognitiva y del Aprendizaje, Facultad Latinoamericana de Ciencias Sociales (FLACSO).

ORCID: https://orcid.org/0000-0002-2719-4963

Autor corresponsal: mery053@hotmail.com

\section{Angel M. Elgier}

Facultad Latinoamericana de Ciencias Sociales, Argentina. Universidad Abierta Interamericana, Facultad de Psicología y Relaciones Humanas, Argentina. Consejo Nacional de Investigaciones Científicas y Técnicas (CONICET), Argentina.

Licenciado en Psicología, Universidad de Buenos Aires (UBA). Doctor en Psicología, Universidad Nacional de Córdoba (UNC). Investigador del Consejo Nacional de Investigaciones Científicas y Técnicas (CONICET).

ORCID: https://orcid.org/0000-0002-6110-5151

angel.elgier@uai.edu.ar

\section{Lucas G. Gago Galvagno}

Facultad Latinoamericana de Ciencias Sociales, Argentina. Universidad Abierta Interamericana, Facultad de Psicología y Relaciones Humanas, Argentina. Consejo Nacional de Investigaciones Científicas y Técnicas (CONICET), Argentina.

Profesor y Licenciado en Psicología, Universidad de Buenos Aires (UBA). Doctor en Psicología, Universidad Católica Argentina (UCA). Becario doctoral del Consejo Nacional de Investigaciones Científicas y Técnicas (CONICET).

ORCID: https://orcid.org/0000-0001-5993-3866

lucas.gagogalvagno@hotmail.com

\section{Alba E. Mustaca}

Universidad Abierta Interamericana, Facultad de Psicología y Relaciones Humanas, Argentina.

Licenciada en Trabajo Social y Psicología, Universidad de Buenos Aires (UBA). Doctora en Psicología, Universidad Nacional de San Luis (UNSL).

ORCID: https://orcid.org/0000-0001-9699-3147

albamustaca@gmail.com 